\title{
Reducing the weak lensing noise for the gravitational wave Hubble diagram using the non-Gaussianity of the magnification distribution
}

\author{
Christopher M. Hirata, ,, Daniel E. Holz, ${ }^{2}$ and Curt Cutler ${ }^{3}$ \\ ${ }^{1}$ Caltech $M / C$ 350-17, Pasadena, CA 91125, USA \\ ${ }^{2}$ Theoretical Division, MS-B227, Los Alamos National Laboratory, Los Alamos, NM 87545, USA \\ ${ }^{3}$ Jet Propulsion Laboratory, M/S 169-327, 4800 Oak Grove Drive, Pasadena, CA 91109, USA
}

(Dated: May 25, 2010)

\begin{abstract}
Gravitational wave sources are a promising cosmological standard candle because their intrinsic luminosities are determined by fundamental physics (and are insensitive to dust extinction). They are, however, affected by weak lensing magnification due to the gravitational lensing from structures along the line of sight. This lensing is a source of uncertainty in the distance determination, even in the limit of perfect standard candle measurements. It is commonly believed that the uncertainty in the distance to an ensemble of gravitational wave sources is limited by the standard deviation of the lensing magnification distribution divided by the square root of the number of sources. Here we show that by exploiting the non-Gaussian nature of the lensing magnification distribution, we can improve this distance determination, typically by a factor of $2-3$; we provide a fitting formula for the effective distance accuracy as a function of redshift for sources where the lensing noise dominates.
\end{abstract}

PACS numbers: $04.30 . \mathrm{Tv}, 95.36 .+\mathrm{x}, 98.62 . \mathrm{Sb}, 98.80 . \mathrm{Es}$

\section{INTRODUCTION}

In 1998 two groups measuring the luminosity distanceredshift relation (the Hubble diagram) from Type Ia supernovae $(\mathrm{SNe})$ reported that the expansion of the Universe was accelerating [1, 2]. This discovery has stimulated a range of efforts to measure the cosmic expansion history and assess whether it is consistent with a cosmological constant or if alternatives such as quintessence are required. The Type Ia SNe continue to provide one of the most valuable constraints [3], due to quality data at a range of redshifts, and the lack of cosmic variance limitations that plague alternatives such as weak gravitational lensing (WL) and baryon-acoustic oscillations (BAO) at low redshift.

The advent of gravitational wave astronomy has prompted interest in gravitational wave (GW) sources as a standard candle. Schutz [4] showed that a gravitational waveform from merging compact objects can be used to measure the distance to the source; a redshift obtained of an electromagnetic counterpart or host galaxy would then allow one to place the GW source on a luminosity distance-redshift relation. The GW source method has the key advantage over other standard candles that its luminosity can be determined from fundamental physics, thus alleviating the common concern with standard candles that they could evolve with cosmic time. They are also insensitive to dust opacity. Finally, many proposed space-based gravitational wave detectors measure test mass separations directly in units of the laser wavelength, as opposed to supernovae, which measure relative distances and require an independent calibration ladder in order to measure absolute distances. Thus GW

*Electronic address: chirata@tapir.caltech.edu sources offer the potential for a low-systematics probe of the expansion history of the Universe. Examples of such sources would include mergers of massive black holes, observable with the Laser Interferometer Space Antenna (LISA [4]]), and binary neutron star or stellar mass black hole binaries, observable with a more futuristic spacebased detector in the $\sim 1 \mathrm{~Hz}$ band such as the Big Bang Observer (BBO). The latter, in particular, could potentially observe hundreds of thousands of neutron starneutron star binary inspirals [5].

A final advantage is that the distance determination to a GW source is limited by the signal-to-noise of the measurement (and by partial degeneracies with binary parameters), as opposed to Type Ia SNe, which have a seemingly random $\sim 15 \%$ scatter in their luminosities even after the light curve stretch correction. This means that the statistical power of GW sources may be interesting even if the number of usable sources is far less than the number of usable SNe. In fact, for high signal-to-noise detections of GW sources, the distance determination should be limited not by the intrinsic dispersion of the source nor by the measurement error, but rather by weak lensing magnification: the intervening matter between us and the source will magnify the GW source and affect our measurement of the distance. The apparent flux from the source is increased by some factor $\mu$, which is usually $\sim 1$, and the apparent distance $D_{\text {app }}$ thus differs from the true distance $D$ according to $D_{\text {app }}=D \mu^{-1 / 2}$. This phenomenon of course occurs for all standard candles, and has long been recognized as an issue for $\mathrm{SNe}$ [6 8], but its importance relative to intrinsic dispersion is much greater for gravitational waves. (Gravitational wave measurements with nearby sources or with lower signal-to-noise per source, such as the proposed binary progenitors of short gamma ray bursts [9, 10], are much less affected since the lensing scatter is subdominant.) 
The usual way of accounting for the magnification effect is to suppose that it adds in quadrature with the intrinsic-luminosity and apparent flux measurement contributions to the distance error. That is,

$$
\sigma_{\ln D^{2}}=\sqrt{\sigma_{\ln L}^{2}+\sigma_{\ln F}^{2}+\sigma_{\mu}^{2}}
$$

where $D$ is the distance, $L$ is the intrinsic luminosity, $F$ is the measured flux, and $\mu$ is the magnification; with $N$ sources, this uncertainty is reduced by a factor of $\sqrt{N}$ [8, 11]. Since the last term dominates for GW sources and is significant for high- $z \mathrm{SNe}$, there is great motivation to try to reduce it. One possibility would be to try to construct an estimated magnification $\hat{\mu}$ from external data sets; the last term should then be replaced by $\sigma^{2}(\mu-\hat{\mu})$. Unfortunately, WL shear maps have too much high spatial frequency noise to be useful as a magnification estimator for point sources [12], but galaxy maps are highly correlated with the mass distribution and may be able to reduce the lensing dispersion term by a factor of $\sim \sqrt{3}[13-15$. Maps of flexion (i.e. the gradient of the shear measured using the banana- or trefoil-shaped distortion of a galaxy [16]) could also be useful if very high source densities $\left(>100 \mathrm{arcmin}^{-2}\right)$ can be obtained [17].

The purpose of this paper is to explore yet another method of reducing the lensing dispersion in Eq. (1). Because the probability density function (PDF) of $\mu$ is highly non-Gaussian (technically non- $\Gamma$, as we show in Appendix (A), our ability to centroid it using $N$ sources can be much better than $\sigma_{\mu} / \sqrt{N}$. This is in fact a familiar result: as an extreme case, many distributions such as the Airy diffraction pattern in optical astronomy can be centroided even though their variances are formally infinite. In the case of lensing magnification, the variance of $\mu$ is often dominated by a long tail to positive values, corresponding to lines of sight that pass through a galaxy or its halo, whereas the information content is dominated by relatively empty lines of sight with $\mu-1$ sharply peaked around a slightly negative value. In such cases, the use of outlier-rejecting statistics not only removes sources with misidentified redshifts [5] but also reduces the uncertainty in $D(z)$ for correctly identified sources. (A similar point has been made in the recent paper by Shang \& Haiman [18].)

This paper is organized as follows. In Section III we discuss the information-theoretic limits on centroiding a distribution. Numerical results and simulations are presented in Section III] Section IV gives cosmological constraints obtainable with the reduced centroid errors for $\mathrm{BBO}$ and for LISA. We conclude and briefly discuss systematics in Section $\mathrm{V}$

We focus here on the problem of measuring $D(z)$ from GW sources. However, the formalism is applicable to any standard candle, and we briefly discuss the implications for Type Ia supernovae.

\section{CENTROIDING A DISTRIBUTION}

In this section, we consider the problem of determining the distance $D$ to a population of $N$ standard sources at some redshift $z$. For simplicity, we consider first the case with no intrinsic dispersion in the source luminosity, and then generalize to the case with a known additional source of scatter (e.g. an intrinsic dispersion or measurement uncertainty). For large $N$, the maximum likelihood estimator for $\ln D^{2}$ is asymptotically unbiased, and saturates the Cramer-Rao bound on the uncertainty given by the Fisher information.

\section{A. No intrinsic dispersion}

We consider a distribution of sources with some magnification probability $P(x)$, where $x=\ln \mu$. The apparent distance to the source is

$$
\ln D_{\text {app }}^{2}=\ln D^{2}-x .
$$

Our job is then straightforward: we are to estimate $\ln D^{2}$ from $N$ independent and identically distributed values of $\ln D_{\text {app }, i}^{2}$. If $N$ is sufficiently large (how large will be investigated below), then we may use the Fisher information to determine how well we can measure $\ln D^{2}$. For a single sample $(N=1)$, the Fisher information is

$$
\begin{aligned}
I_{\ln D^{2}}^{(1)} & =\left\langle\left[\frac{d}{d \ln D^{2}} \ln P\left(\ln D^{2}-\ln D_{\text {app }}^{2}\right)\right]^{2}\right\rangle \\
& =\left\langle\left[\frac{d}{d x} \ln P(x)\right]^{2}\right\rangle \\
& =\int P(x)\left[\frac{d}{d x} \ln P(x)\right]^{2} d x .
\end{aligned}
$$

For multiple independent samples, the Fisher matrix simply adds so that $I_{\ln D^{2}}=N I_{\ln D^{2}}^{(1)}$. For large $N$, the uncertainty in $\ln D^{2}$ would then be

$$
\sigma\left(\ln \hat{D}^{2}\right) \approx \frac{1}{\sqrt{N I_{\ln D^{2}}^{(1)}}} .
$$

\section{B. Intrinsic dispersions and measurement uncertainties}

We now consider the case where the error $x$ in $\ln D_{\text {app }}^{2}$ is determined not just by lensing, but also by an additional contribution such as intrinsic dispersion (for statistical standard candles such as supernovae) or flux measurement uncertainty (which exists for any standard candle). We denote the lensing contribution by $x_{1}$ and the additional dispersion by $x_{2}$. We assume these to be independent with probability distributions $P_{1}$ and $P_{2}$, so 
that the probability of $x$ is given by a convolution:

$$
P(x)=\int P_{1}\left(x_{1}\right) P_{2}\left(x-x_{1}\right) d x_{1} .
$$

This assumption should be true for the case where $x_{2}$ is dominated by intrinsic dispersion, since the intrinsic luminosity scatter of a source physically cannot depend on the lens alignment. It might be violated for the case of the measurement uncertainty since a magnified source will be detected at higher $S / N$ and thus is likely to have a smaller fractional error on the flux; however this is probably only significant for the strongly lensed sources, which do not dominate the information integral, Eq. (3).

In this paper, the intrinsic dispersion/measurement uncertainty will be taken to be a lognormal distribution:

$$
P\left(x_{2}\right)=\frac{1}{\sqrt{2 \pi} \sigma_{x_{2}}} \exp \left[-\frac{\left(x_{2}+\sigma_{x_{2}}^{2} / 2\right)^{2}}{2 \sigma_{x_{2}}^{2}}\right],
$$

with the offset $-\sigma_{x_{2}}^{2} / 2$ designed to ensure $\left\langle e^{x_{2}}\right\rangle=1$.

The Fisher information for the convolved distribution, and for its improvement ratio, can be obtained from the usual formula, Eq. (3).

\section{Estimators}

In the limit of large $N$, the maximum likelihood estimator for $\ln D^{2}$ achieves the Fisher information errors. This estimator is given by the implicit equation

$$
\sum_{i=1}^{N} w\left(\ln D_{\text {app }, i}^{2}-\ln \hat{D}^{2}\right)=0,
$$

where

$$
w(x)=-\frac{d}{d x} \ln P(x)
$$

is a weight function.

This can be compared to the "conventional" estimator based on flux-averaging [8], i.e. based on conservation of mean surface brightness $\langle\mu\rangle=1$, which implies $\left\langle D_{\text {app }}^{-2}\right\rangle=$ $D^{-2}$. This approach gives another distance estimate,

$$
\hat{D}_{\mathrm{C}}^{-2}=\frac{1}{N} \sum_{i=1}^{N} D_{\mathrm{app}, i}^{-2}
$$

where the subscript "C" is used to denote the conventional estimator. Note that Eq. (9) is model-independent in the sense that no functional form for the magnification $\mathrm{PDF}$ is assumed. Since $D_{\mathrm{app}, i}^{-2}=D^{-2} \mu$, the standard deviation of Eq. (9) is $D^{-2} \sigma_{\mu}$, and the logarithmic uncertainty is

$$
\sigma\left(\ln \hat{D}_{\mathrm{C}}^{2}\right) \approx \frac{\sigma_{\mu}}{\sqrt{N}}
$$

The Fisher error, $\left[N I_{\ln D^{2}}^{(1)}\right]^{-1 / 2}$, is of course always less than or equal to Eq. (10). We give an elementary proof of this inequality in Appendix A. There we also show that equality holds only in the case where the magnification PDF is a $\Gamma$-distribution, Eq. (A2). We expect that in practice Eq. (4) should be a substantial improvement over Eq. (10) because the $\Gamma$-distribution does not resemble a realistic magnification $\mathrm{PDF}$, since it cuts off exponentially at large magnifications. The $\Gamma$ distribution is also far more symmetric than "real" PDFs: it always has a normalized skewness

$$
S_{3}^{\prime}=\frac{\left\langle(\mu-1)^{3}\right\rangle}{\left\langle(\mu-1)^{2}\right\rangle^{2}}
$$

equal to $S_{3}^{\prime}=2$.

\section{NUMERICAL RESULTS}

We have now completed our survey of the theory; it is now time to actually evaluate the Fisher information for realistic magnification PDFs. We first describe the construction of the lensing magnification PDFs and then display results. Finally we simulate the effect of a finite number of sources on the maximum likelihood estimator.

\section{A. Lensing PDFs}

We use the stochastic universe method presented in Holz \& Wald [7] to calculate the lensing PDFs. This method calculates the full (weak and strong) lensing distributions utilizing a Monte Carlo code: the universe in the vicinity of a photon path is generated randomly, and the lensing effects from the matter distribution are calculated analytically. We approximate the matter in the universe as pure dark matter smoothly distributed in NFW halos [19], with the halo masses drawn from the Sheth-Tormen mass function [20], and with cosmological parameters $\Omega_{m}=0.28, \Omega_{\Lambda}=0.72, h=0.7$, and $\sigma_{8}=0.79$. The lensing distributions are relatively insensitive to both the details of the lenses and the values of the cosmological parameters [21].

The Fisher analysis requires that the magnification PDF be smooth, since Monte Carlo noise results in a spurious, positive definite contribution to Eq. (3). We have used several versions of the smoothing procedure. The default procedure (used for most of the results in this paper unless otherwise specified) is to bin the lensing PDF in bins of width $\Delta \mu=10^{-3}$. Then a triangle-hat smoothing kernel is used, i.e.

$$
P_{\text {smooth }}(\mu)=\frac{1}{(S+1)^{2}} \sum_{j=-S}^{S}(S+1-|j|) P(\mu+j \Delta \mu) \text {. }
$$

Since more smoothing is needed in the tail of the distribution than the peak, we generate two smoothed distributions $P_{1}$ and $P_{2}$ with different values of the smoothing 


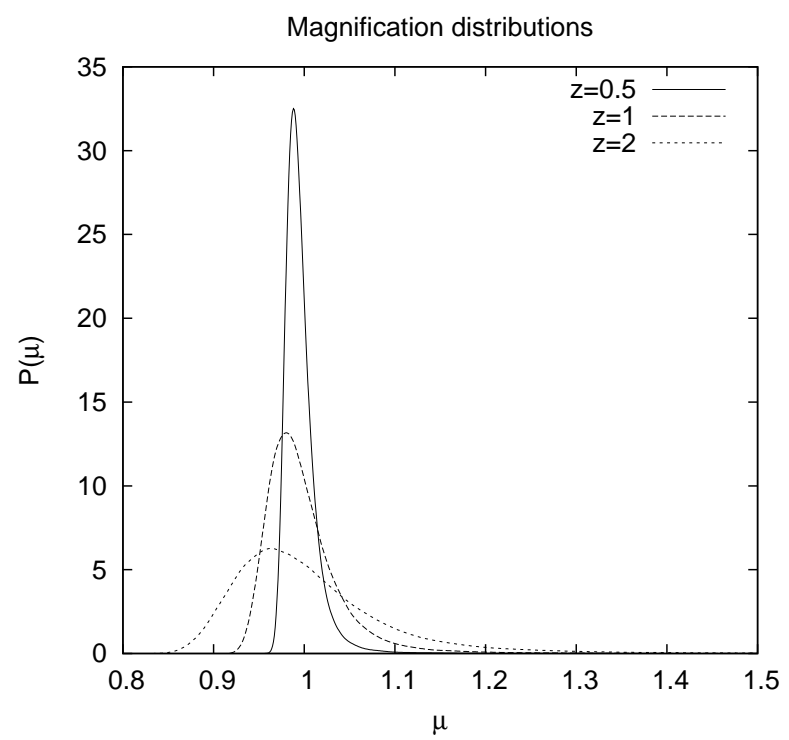

FIG. 1: The magnification distributions $P(\mu)$ at $z=0.5,1$, and 2 .

$S_{1}$ and $S_{2}$. The distributions are combined according to

$$
P_{\text {smooth }}(\mu)=\frac{P_{2}+c P_{1}}{1+c}
$$

where $c=e^{50(\mu-1.03)}$. This results in an effective smoothing of $P_{1}$ at large $\mu$ and $P_{2}$ at small $\mu$ with smooth interpolation. For $z=0.5$ we choose $\left(S_{1}, S_{2}\right)=(2,15)$; for $z=1,(5,20)$; and for $z=2,(10,30)$. The Monte Carlo PDF is generated only out to $\mu=2$; above this, we assume a $P(\mu) \propto \mu^{-3}$ scaling as appropriate for large magnifications (near a caustic). This matters little since this region contributes little to Eq. (31). The smoothed distributions at $z=0.5,1$, and 2 are shown in Figure 1 .

We have tried other methods of smoothing to ensure robustness. For example, we have tried re-computing the Fisher integral, Eq. (3), by fitting $P(\mu)$ with least-squares quadratic functions in intervals of width $\Delta \mu=0.02$ (at $\mu>1+0.04 z$ ) or 0.01 (at $\mu \leq 1+0.04 z$ ), and using the fit to analytically compute $d P / d x$. The integral is chopped off at the 0.1-percentile point of the distribution to avoid spurious effects (such as fits that pass through zero) since the quadratic polynomial is not a good approximation near the minimum value of $\mu$. This procedure led to uncertainties that differed by at most $6 \%$ from our fiducial smoothing procedure.

For completeness, we have also utilized a SavitzkyGolay smoothing filter, with width $\Delta \mu=0.05$, and have found results differing by $\sim 4 \%$ from our fiducial smoothing.

\section{B. Fisher results}

In Figure 2, we show the Fisher matrix error per source, $\left[I_{\ln D^{2}}^{(1)}\right]^{-1 / 2}$, as well as the flux-averaging error,

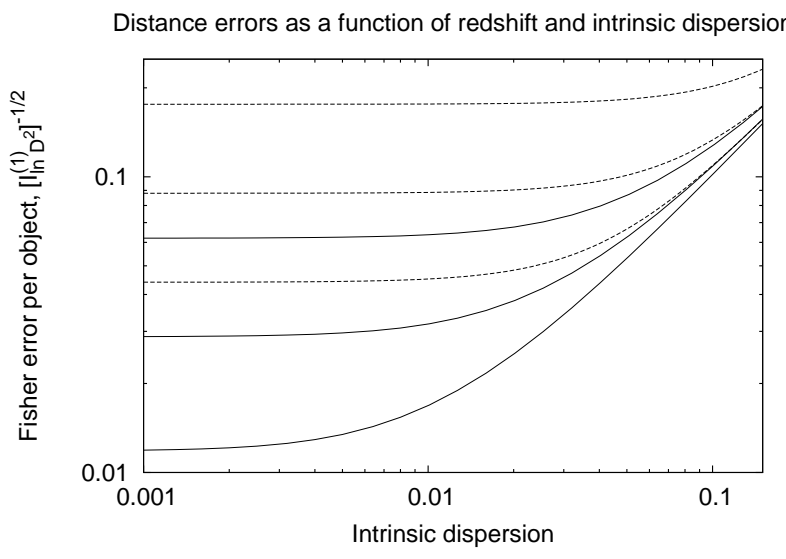

FIG. 2: The solid curves show the Fisher matrix error per source, $\left[I_{\ln D^{2}}^{(1)}\right]^{-1 / 2}$, as a function of intrinsic dispersion $\sigma_{x_{2}}$ for source redshifts of 0.5 (bottom), 1.0, and 2.0 (top). The dashed curves show the error per source using the fluxaveraging approach, Eq. (1).

Eq. (1). For the flux-averaging error, we have used the approximation $\sigma_{\mu} \approx 0.088 z[8]$. The results are shown for 3 redshifts, $z=0.5,1.0$, and 2.0, and for a range of intrinsic dispersions $\sigma_{x_{2}}$. For large intrinsic dispersion, lensing adds negligible additional dispersion and the error per source is $\sigma_{x_{2}}$. For small $\sigma_{x_{2}}$, the lensing dispersion dominates, and we see that the Fisher matrix errors (solid curves) are factors of 2-3 below the flux-averaging errors (dashed lines).

In the case of the flux-averaging method, the variance per source is as noted above given by the quadrature sum,

$$
\sigma_{\ln D^{2}}^{2}=\sigma_{\ln D^{2}}^{2}\left(\sigma_{x_{2}}=0\right)+\sigma_{x_{2}}^{2} .
$$

In the case of the centroiding method, no such exact relation holds. However, it turns out that the quadrature sum approximation

$$
\left[I_{\ln D^{2}}^{(1)}\right]^{-1} \approx\left[I_{\ln D^{2}}^{(1)}\left(\sigma_{x_{2}}=0\right)\right]^{-1}+\sigma_{x_{2}}^{2}
$$

has an error of at most $10 \%$ over the range of redshifts $z \geq 0.5$ probed (the maximum error is at low redshift where the magnification PDF is most non-Gaussian). By construction, Eq. (15) is exact when one or the other source of error dominates.

For an intrinsic scatter $\sigma_{x_{2}} \approx 0.15$ appropriate to Type Ia supernovae, the intrinsic scatter is dominant over the lensing even for the flux-averaging method for $z \leq 1.7$. At $z=1.7$ the centroiding method reduces the effective error per source $\left[I_{\ln D^{2}}^{(1)}\right]^{-1 / 2}$ from 0.22 to 0.16 , which is a modest improvement but not nearly what one finds for low dispersion gravitational wave sources.

\section{Finite sample size}

The maximum likelihood estimator is known to achieve the Fisher uncertainty only in the limit of large numbers 
of sources. For a finite number of sources per redshift bin, Eq. (7) may be biased and may have a larger error than the Fisher information would suggest. The bias in the estimator for $\ln D^{2}$ can be computed and subtracted using Monte Carlo simulations, assuming that $P(\mu)$ is known. However, the uncertainty in $\ln D^{2}$ may be larger than the Fisher estimate. Here we use Monte Carlo simulations to measure the scatter in $\ln \hat{D}^{2}$, and show that for $N \geq 4$ sources the Fisher estimate is accurate to within $\leq 10 \%$.

We have constructed our Monte Carlo simulations for any source redshift $z$ and intrinsic dispersion $\sigma_{x_{2}}$ by first drawing $N$ random deviates from the $P(\mu)$ distribution. For each $\mu_{i}$, we obtain an estimated luminosity distance $D_{i}^{2}=D^{2} \mu_{i}^{-1}$. Then we maximize

$$
\ln L(\hat{D})=\sum_{i=1}^{N} P\left(x=\ln \hat{D}^{2}-\ln D_{i}^{2}\right) .
$$

Then $q=\ln \hat{D}^{2}-\ln D^{2}$ has a probability distribution that depends only on $N$ and $P(\mu)$ ( $D$ trivially drops out). A $95 \%$ confidence interval on $\ln D^{2}$ can be obtained by taking $\ln \hat{D}^{2}$ and subtracting the 2.5 th or 97.5 th percentiles of the distribution of $q$, i.e. at $95 \%$ confidence

$$
\ln \hat{D}^{2}-q_{0.975}<\ln D^{2}<\ln \hat{D}^{2}-q_{0.025},
$$

where $q_{\alpha}$ is defined by $\int_{-\infty}^{q_{\alpha}} P(q) d q=\alpha$. The width of the confidence interval is $q_{0.975}-q_{0.025}$.

In comparison, the usual assumption of Gaussian errors with width given by the Fisher calculation would suggest that the width of the $95 \%$ confidence interval would be $2 \cdot 1.960\left[N I_{\ln D^{2}}^{(1)}\right]^{-1 / 2}$, where $95 \%$ of the probability in a unit normal distribution lies between \pm 1.960 .

The widths resulting from the full Monte Carlo procedure are compared with the Fisher calculation in Figure 3. As expected, the simulated errors are larger than the Fisher prediction. However the discrepancy drops rapidly for $N \geq 4$. This is because even 4 events are usually sufficient to identify and reject the strongly magnified sources.

One would intuitively expect that the Fisher errors are approached more rapidly in the presence of intrinsic dispersion because this results in a $P(x)$ that is more nearly Gaussian. Indeed, this is what we find in simulations. For example, at $z=1$ and $N=4$, we find that with no intrinsic dispersion the $99 \%$ confidence region is 1.12 times wider than the Fisher calculation suggests. This factor drops to 1.09 if we impose an intrinsic dispersion of $\sigma_{x_{2}}=0.02$, to 1.07 at $\sigma_{x_{2}}=0.05$, and 1.04 at $\sigma_{x_{2}}=0.1$.

\section{COSMOLOGICAL CONSTRAINTS}

We now consider the possible cosmological constraints from gravitational wave sources. We begin by describing our methodology for computing parameter constraints (Sec. IVA). We then turn to two specific examples: BBO (Sec. IVB) and LISA (Sec. IVC).

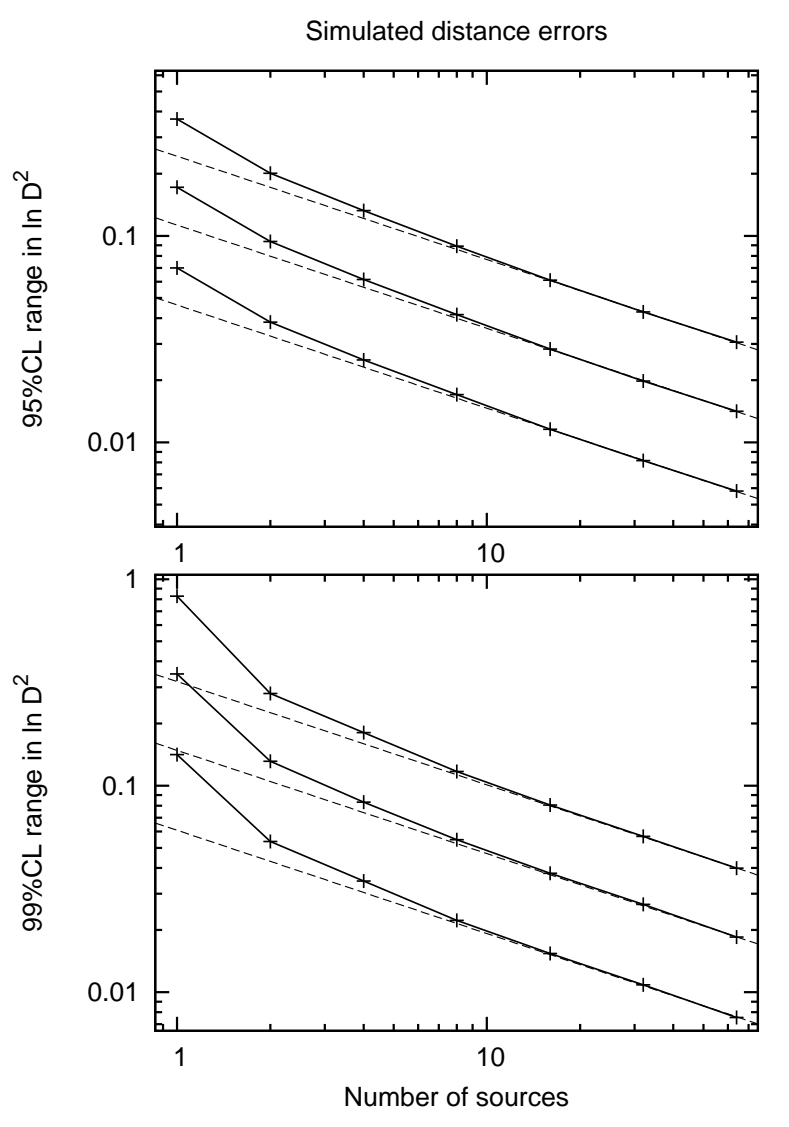

FIG. 3: The full width of the $95 \%$ and $99 \%$ confidence ranges for $\ln D^{2}$ for source redshifts of 0.5 (bottom curve), 1.0 , and 2.0 (top curve), as a function of the number of sources $N$. Zero intrinsic dispersion is assumed. The points are the results from Monte Carlo simulations. The dashed lines are the Fisher predictions assuming Gaussian errors, i.e. $2 \cdot 1.960\left[N I_{\ln D^{2}}^{(1)}\right]^{-1 / 2}$ and $2 \cdot 2.576\left[N I_{\ln D^{2}}^{(1)}\right]^{-1 / 2}$ respectively. Note the good agreement of the Monte Carlo and Fisher results for $N \geq 4$. This plot used $10^{4}$ simulations.

\section{A. Forecasting methodology}

It is straightforward to generalize Eq. (3) to $N$ sources, at a range of range of redshifts $\left(z_{1}, z_{2}, \cdots, z_{N}\right)$, and to a cosmological model depending on $N_{P}$ parameters. We denote the cosmological parameters by $\left\{\lambda^{\alpha}\right\}_{\alpha=1}^{N_{P}}$. Let $D_{\text {app }, i}$ represent the $N$ measured luminosity-distances, and let $x_{i} \equiv\left(\ln D\left(z_{i}\right)^{2}-\ln D_{\text {app,i }}^{2}\right)$. The magnifications for each source should be very close to statistically independent, since a gravitational wave detector sees the whole sky. (Note that this is unlike the case of a supernova survey with an optical telescope, which inherently has a narrow field of view and hence depending on the survey strategy may suffer from correlated magnifications 
[22].) Thus the $N_{P}$-dimensional Fisher matrix is

$$
\begin{aligned}
I_{\alpha \beta}= & \int P\left(x_{1}\right) P\left(x_{2}\right) \cdots P\left(x_{N}\right) \\
& {\left[\left(\sum_{i=1}^{N} \frac{\partial x_{i}}{\partial \lambda^{\alpha}} \frac{d \ln P\left(x_{i}\right)}{d x_{i}}\right)\right.} \\
& \left.\times\left(\sum_{j=1}^{N} \frac{\partial x_{j}}{\partial \lambda^{\beta}} \frac{d \ln P\left(x_{j}\right)}{d x_{j}}\right)\right] d x_{1} \cdots d x_{N},
\end{aligned}
$$

where we have used the fact that $P\left(x_{1}, x_{2}, \cdots, x_{N}\right)=$ $P\left(x_{1}\right) P\left(x_{2}\right) \cdots P\left(x_{N}\right)$, since the $N$ measurements are independent. Using the fact that $\int P\left(x_{i}\right) d x_{i}=1$ and hence that $\int P\left(x_{i}\right)\left[\partial \ln P\left(x_{i}\right) / \partial \lambda^{\alpha}\right] d x_{i}=0$, it is easy to see in the double-sum over $i$ and $j$, the terms with $i \neq j$ are all zero. Hence our expression for $I_{\alpha \beta}$ reduces to

$$
I_{\alpha \beta}=\sum_{i=1}^{N} \int P\left(x_{i}\right)\left[\frac{d \ln P\left(x_{i}\right)}{d x_{i}}\right]^{2} \frac{\partial x_{i}}{\partial \lambda^{\alpha}} \frac{\partial x_{i}}{\partial \lambda^{\beta}} d x_{i} .
$$

Of course, $\partial x_{i} / \partial \lambda^{\alpha}$ is just $\partial\left(\ln D_{i}^{2}\right) / \partial \lambda^{\alpha}$. Thus we arrive at our final expression for the Fisher matrix:

$$
I_{\alpha \beta}=\sum_{i=1}^{N} I_{\ln D^{2}}^{(1)}\left(z_{i}\right)\left[\frac{\partial\left(\ln D^{2}\left(z_{i}\right)\right)}{\partial \lambda^{\alpha}} \frac{\partial\left(\ln D^{2}\left(z_{i}\right)\right)}{\partial \lambda^{\beta}}\right] .
$$

The information for a single source $I_{\ln D^{2}}^{(1)}\left(z_{i}\right)$ is simply the integral, Eq. (3), where the probability distribution for $x$ contains lensing noise and (if significant) measurement noise as well.

To rapidly estimate $I_{\ln D^{2}}^{(1)}(z)$ for any $z$ (up to $z=3$ ), we (i) calculated the magnification distribution $P_{z}(\mu)$, for redshifts $z \in\{0.5,1,1.5,2,2.5,3\}$ using the method of Holz \& Wald [7], (ii) used (smoothed versions of) these distributions to calculate $I_{\ln D^{2}}^{(1)}(z)$ for these 6 redshifts, using Eq. (3), and then (iii) fit these results to a smooth curve that has the correct asymptotics (going to 0 as $z \rightarrow 0$ and going to a constant as $z \rightarrow \infty$ ). We find the following to be a suitable fit:

$$
\left[I_{\ln D^{2}}^{(1)}(z)\right]^{-1 / 2}=C\left[\frac{1-(1+z)^{-\beta}}{\beta}\right]^{\alpha},
$$

where $C=0.066, \beta=0.25$, and $\alpha=1.8$. This function is shown in Figure 4. Note that we have not explored its validity at $z>3$.

At low redshifts, the peculiar velocity error dominates; assuming a width of $300 \mathrm{~km} \mathrm{~s}^{-1}$ (e.g. 23]) gives an additional contribution to $\sigma_{x_{2}}$ of twice $300 \mathrm{~km} \mathrm{~s}^{-1}$ divided by the Hubble velocity $c z$, which is $0.002 z^{-1}$. We have approximated this error by a quadrature-sum with the lensing + measurement noise, which our tests of Eq. (15) suggest will not be in serious error.

In some cases, a very large number of sources will be observed (possibly of order $10^{5}$ for BBO). In such cases, it is appropriate to bin the sources into redshift slices

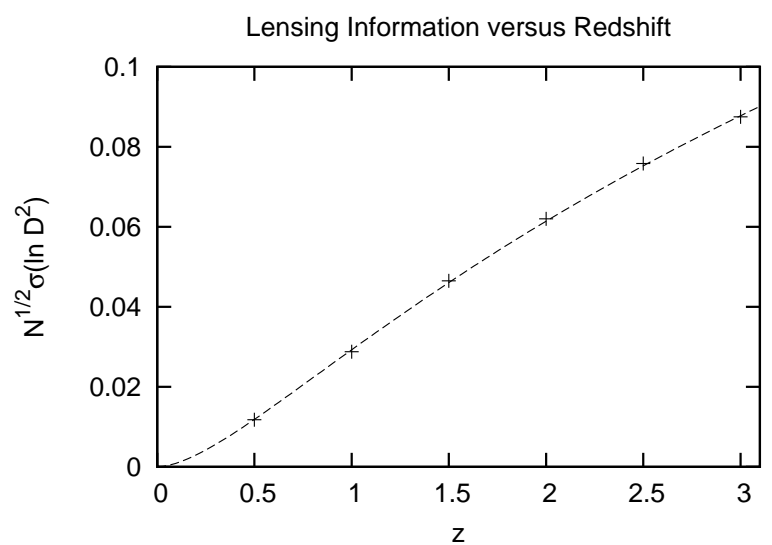

FIG. 4: The uncertainty per source on the Hubble plot, $\left[I_{\ln D^{2}}^{(1)}(z)\right]^{-1 / 2}$, as a function of redshift. The points are obtained from evaluation of the information integral over $P(\mu)$, while the curve is the fit of Eq. (22).

as is often done for supernova forecasts [24]. Given a redshift distribution $\Pi(z)$, we bin sources into redshift slices of width $\Delta z=0.1$, and the number of sources in each bin is computed according to $N_{i}=N_{\mathrm{src}} \Pi(z) \Delta z$. We have cut off the bins at redshifts below $z_{\min }$, where $z_{\min }$ is defined such that we expect 1 source at $z<z_{\min }$, i.e. $\int_{0}^{z_{\min }} \Pi(z) d z=N_{\mathrm{src}}^{-1}$. This is to prevent a mission that collects a small number of low- $z$ sources from taking advantage of a highly precise "constraint" obtained locally $(z \ll 1)$ from e.g. 0.01 sources with ultra-precise distances.

Our parameter space $\left\{\lambda^{\alpha}\right\}$ includes the present-day densities of baryons $\Omega_{\mathrm{b}} h^{2}$, matter $\Omega_{\mathrm{m}} h^{2}$, and dark energy $\Omega_{\mathrm{de}} h^{2}$, as well as the spatial curvature $\Omega_{\mathrm{K}} h^{2}$. The equation of state (pressure:density ratio) of the dark energy is described by the 2-parameter model

$$
w_{\mathrm{de}}(a) \equiv \frac{P_{\mathrm{de}}(a)}{\rho_{\mathrm{de}}(a)}=w_{0}+w_{a}(1-a),
$$

where the parameters are $\left(w_{0}, w_{a}\right)$ and the fiducial "cosmological constant" model has $w_{0}=-1$ and $w_{a}=0$. We also include the primordial spectrum of Gaussian adiabatic cosmological perturbations, assumed to be a power law (2 parameters: amplitude and spectral index), which are required when combining GW data with the CMB or weak lensing; and the absolute magnitude of a Type Ia supernova, required when including the supernova Hubble diagram. This gives a 9-dimensional parameter space. Note that the Hubble constant $H_{0}$ is not an additional parameter since it is determined by $\Omega_{\mathrm{m}} h^{2}, \Omega_{\mathrm{de}} h^{2}$, and $\Omega_{\mathrm{K}} h^{2}$.

We run our forecasts both internal to the GW method, and in combination with other methods of probing cosmology; the latter cases include the "Stage III" (i.e. nearterm ground based) results for supernovae, weak lensing, and baryon-acoustic oscillations, and the Planck CMB constraints, as forecast by the Figure of Merit Science Working Group (FoMSWG) [24]. 
We compute the Figure of Merit (FoM) defined by the Dark Energy Task Force (DETF) 25] for several combinations of future data sets. This FoM is defined to be proportional to the inverse-area of the error ellipse in the $\left(w_{0}, w_{a}\right)$ plane, i.e.

$$
\mathrm{FoM}_{\mathrm{DETF}} \equiv \frac{1}{\sigma\left(w_{0}\right) \sigma\left(w_{a}\right) \sqrt{1-\rho^{2}\left(w_{0}, w_{a}\right)}}
$$

where $\rho\left(w_{0}, w_{a}\right)$ is the correlation coefficient. The DETF Figure of Merit is not a unique (or even necessarily the best) way to present the performance of a dark energy experiment - see the discussion in the FoMSWG report [24] - but it is well suited to our objective here, which is to show that our magnification PDF centroiding method leads to significantly tighter dark energy constraints from GW sources.

\section{B. Example: BBO}

We consider a population of sources with the redshift distribution of Ref. [5], appropriate to neutron starneutron star (NS-NS) binaries. Two cases are considered for the error of the distance determination: an ideal case (IDEAL, $\sigma_{x_{2}}=0$ ), and an error of $1.4 z \%$ (NSNS, $\sigma_{x_{2}}=0.028 z$ ), with the latter appropriate to BBO parameter forecasts [5]. For each of these cases, we consider two subcases for the distance determination, the flux-averaging method (AVE) and the centroiding method described here (CEN). For the centroiding case, we used the method described above, while for the flux-averaging cases, the $\ln D^{2}$ uncertainties per source are $0.088 z$ (IDEAL.AVE) or $\sqrt{0.088^{2}+0.028^{2}} z$ (NSNS.AVE).

In Figure 5, we show the DETF Figure of Merit for the combined constraints. We have not included any systematics in the GW constraints. This is of course an optimistic case, and it is not yet clear whether the systematic errors can be made negligible for a BBO-class mission. For example, while the physics of the GW source (the 2body problem in general relativity) is "clean," there are possible systematic errors associated with (i) the theoretical predictions for the magnification $\operatorname{PDF} P(\mu)$, particulary associated with baryonic physics on small scales; and (ii) the strain calibration of a BBO-type detector [5].

We can see that an improvement of a factor of $\sim 2$ in the FoM is possible with $\sim 130$ well-measured sources, and an order of magnitude improvement (comparable to that promised by various Stage IV projects such as the more grandiose versions of JDEM [42]) is possible with $\sim 1500$ sources. We also see that using the flux averaging rather than the centroiding results in a factor of $\sim 5$ increase in the number of sources required to reach a given DETF FoM for the IDEAL case (and a factor of $\sim 3$ for the NS-NS case).
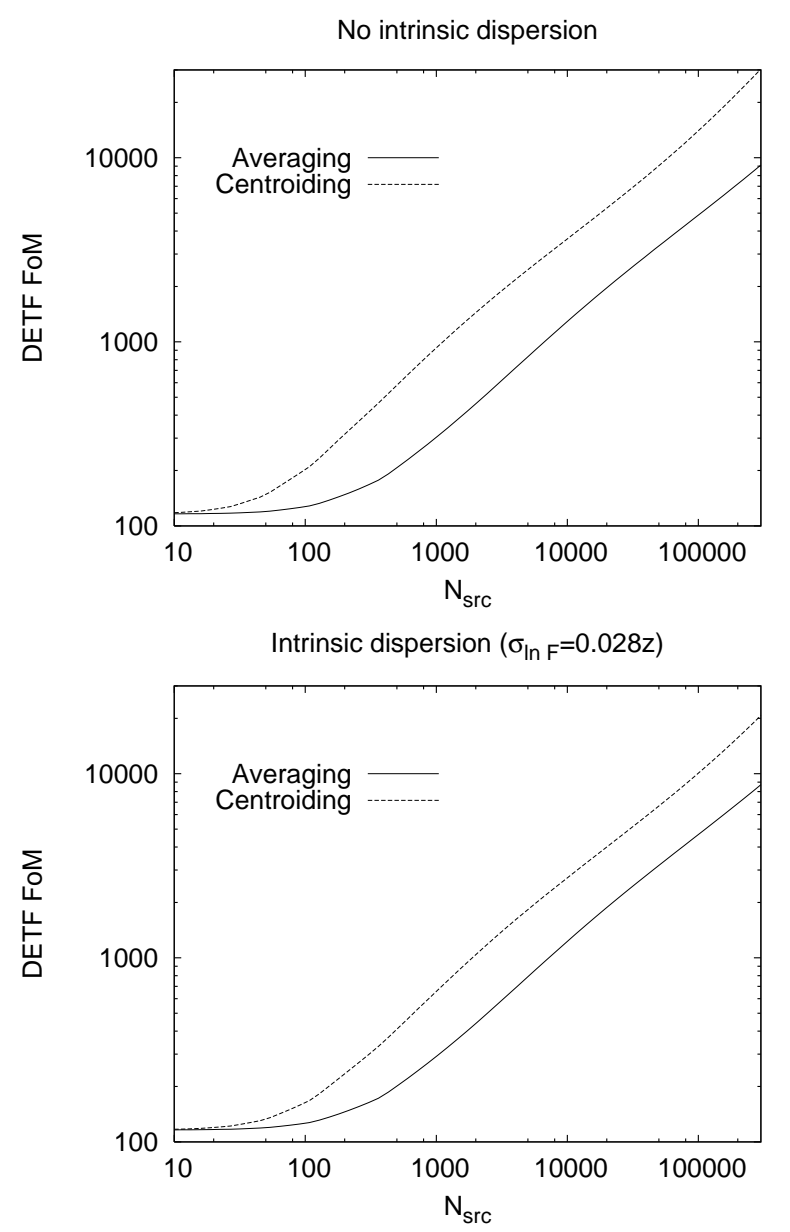

FIG. 5: The DETF FoM as a function of the number of gravitational wave sources $N_{\text {src }}$ used. We also include both the Planck mission and next-generation ground-based dark energy projects (Stage III). The highest $N_{\text {src }}$ value plotted corresponds to $N_{\mathrm{src}}=3 \times 10^{5}$, the rough number expected for $\mathrm{BBO}$.

\section{Example: LISA}

As a second application of the main ideas in this paper, we consider how well cosmological parameters might be constrained by LISA observations of coalescing massive black hole binaries (MBHBs). This question has been considered by several authors, including 11,26 29], with the importance of weak lensing as the dominant effect in limiting LISA's distance-measurement accuracy first being stressed by Hughes \& Holz [30]. The main result of this paper - that previous analyses considerably underestimated the improvement in $D_{L}$-accuracy that comes from combining several measurements - suggests a reexamination of the LISA case.

To provide some context: LISA will be capable of detecting merging MBHBs with masses in the range $\sim 10^{3}-10^{6} M_{\odot}$ out to $z \sim 20$. Estimates of MBHB merger rates vary by several orders of magnitude, depending mostly on the fraction of dark-matter halos that 
have MBHs in their cores at redshifts $z \sim 10-20$. For example, merger-tree models due to Volonteri predict that LISA should detect $\sim 30 \mathrm{MBHBs} / \mathrm{yr}$, mostly at high redshift $(z>4)$ 31]. LISA's angular resolution will typically be a few degrees or worse, so to uniquely identify the host galaxy will generally require some corresponding electromagnetic outburst. Several possible mechanisms for generating outbursts have been explored, including (i) excitation of a shared accretion disk due to the rapid mass loss and/or velocity kick when the binary merges (a consequence of the energy and momentum carried off in GWs) [32-34], (ii) a steep rise in the accretion rate starting months to years after the merger [35], and (iii) a pre-merger burst due to shepherding of the disk around one of the progenitor black holes [36]. But for accurate knowledge of the intensity, spectrum, and time-profile of such electromagnetic outbursts, we may well have to wait until LISA flies. Lacking robust predictions regarding electromagnetic outbursts, the LISA community has tentatively adopted the criterion that an MBHB merger is promising for precise localization if the LISA $1 \sigma$ error ellipse on the sky is $\lesssim 10 \mathrm{deg}^{2}$, which is roughly the field of view of the planned Large Synoptic Survey Telescope (LSST) [43]. Applying this criterion to Volonteri's population models, for example, one finds that $\sim \frac{1}{3}$ of LISA's detected MBHBs could be positioned to within $\lesssim 10$ sq. degrees. Even if only $\sim 20 \%$ of these "promising" events could actually have their redshift determined, this would still lead to of order 10 points on the $D_{L}-z$ curve where the luminosity distances follow from fundamental physics (the 2-body problem in GR). Errors in $D_{L}$ due to noise (both instrumental noise and the confusion background from $\sim 3 \times 10^{8}$ compact galactic binaries) will typically be only $\sim 1-2 \%$, even before incorporating the extremely accurate sky-localization provided by an EM counterpart. Using the precise EM localization will typically reduce this uncertainty by a factor $\sim 2[29]$. Therefore we are in a regime where WL magnifications strongly dominate the $D_{L}$ errors.

Given the large uncertainties, in this paper we adopt a very simple model for the $z$-distribution of localizable sources, which is as follows. We take the rate of MBHB mergers (per unit comoving volume, per unit proper time) to be some constant $\dot{n}$, with the universe evolving according to a flat $\Lambda$ CDM model, with our fiducial values $\left(\Omega_{\Lambda}=0.744, \Omega_{m}=0.256\right)$. Then the redshift distribution of the of the binary sources whose GWs are arriving at LISA (over an observation time $T_{\mathrm{obs}}$ ) is:

$$
\frac{d N}{d z}=4 \pi \dot{n} T_{\mathrm{obs}} \frac{r^{2}(z)}{(1+z) H(z)},
$$

where $r(z)$ is the comoving distance to redshift $z$ and $H(z)$ is the Hubble rate.

We restrict attention to mergers at $z<z_{\max }=3$, and we assume that some constant fraction $F$ of all mergers in this redshift range can be associated with an EM outburst that identifies the host galaxy. The rough justification for limiting our attention to redshifts $z<z_{\max }$ is that as $z$ increases it clearly becomes harder to identify a counterpart, both because the GW SNR is lower (which increases the size of the error box) and because any EM outburst is fainter (and at $z \gtrsim 7.5$ is completely obscured in the LSST bandpasses by intergalactic Lyman- $\alpha$ absorption); we crudely model this fall-off by a Heaviside function. We generate parameter constraints by the method described in Section IVA.

The resulting parameter constraints are shown in Figure 6 for both the case of 10 and 30 usable electromagnetic counterparts. The addition of the GW constraint does not significantly improve the Stage III DETF Figure of Merit - for 30 sources it raises it from 116 to 130 (although we note that the investigation of GW dark energy constraints is still in its early days and further improvements may be possible). However, one may assess the robustness of an overall dark energy program in part by examining how well one can do with each dark energy technique 25]. We therefore show in Figure 6 constraints for the $\mathrm{CMB}+\mathrm{SN}+\mathrm{GW}, \mathrm{CMB}+\mathrm{WL}+\mathrm{GW}$, and $\mathrm{CMB}+\mathrm{BAO}+\mathrm{GW}$ cases. The gravitational wave constraints make large improvements when combined with the supernovae (they partially break the $w_{a}-\Omega_{\mathrm{K}}$ degeneracy by extending the Hubble diagram to higher redshifts) and weak lensing. Less improvement is seen with the BAO model because the BAO already provide some distance constraints in the $z>1$ range. As one can see by comparing the top and bottom rows of the figure, the parameter constraint improvements are much more impressive when using the centroiding than the flux-averaging method.

\section{DISCUSSION}

The luminosity distance-redshift relation is one of the oldest and most fundamental probes of cosmology, and future gravitational wave detectors offer the possibility of measuring it accurately using binary inspirals whose luminosity can be calculated directly from measured parameters and fundamental physics. These sources are however affected by weak gravitational lensing by intervening inhomogeneities in the cosmic mass distribution. This introduces changes of typically a few percent (but occasionally much larger) in the flux, while not significantly affecting the redshift, and thus provides a source of noise in the $D(z)$ relation. We have shown in this paper that exploiting the full power of the likelihood function can reduce this noise: the noise in the $D(z)$ relation is not limited by the lensing dispersion divided by the square root of the number of sources, but rather can be less by a factor of 2-3 if one centroids the distribution of apparent distances using the known non-Gaussian form of the lensing magnification PDF.

We have not discussed here the systematic errors associated with using large numbers of gravitational wave sources for precision low-redshift cosmology, as suggested for BBO. While the signal itself is expected to be clean, 

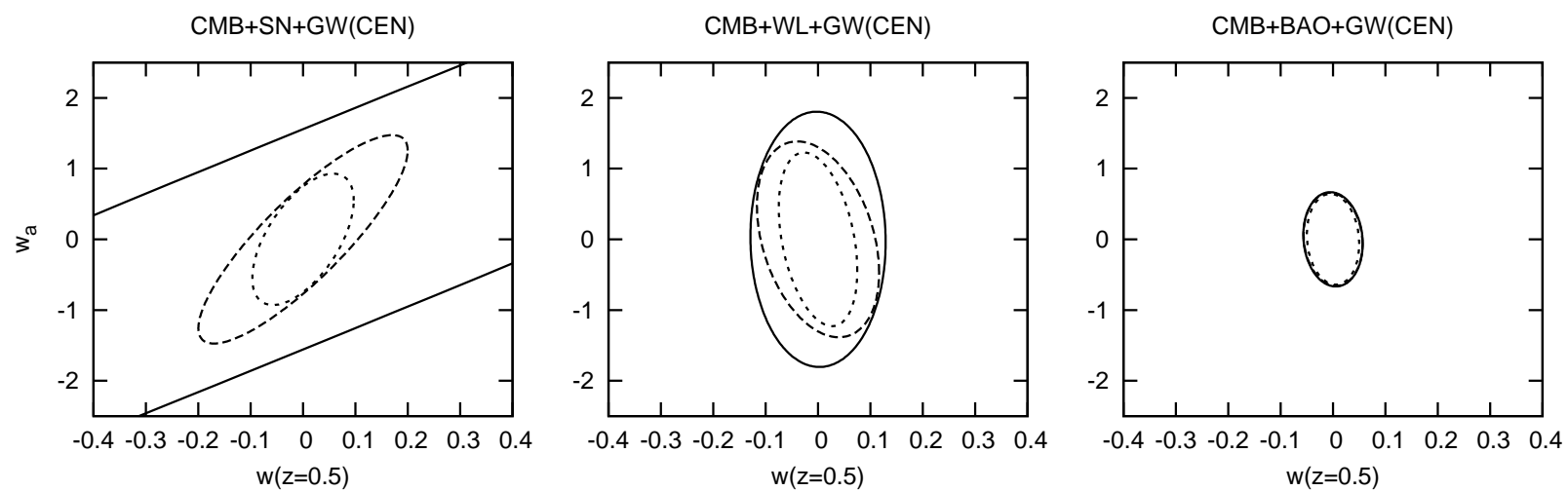

$\mathrm{CMB}+\mathrm{SN}+\mathrm{GW}(\mathrm{AVE})$

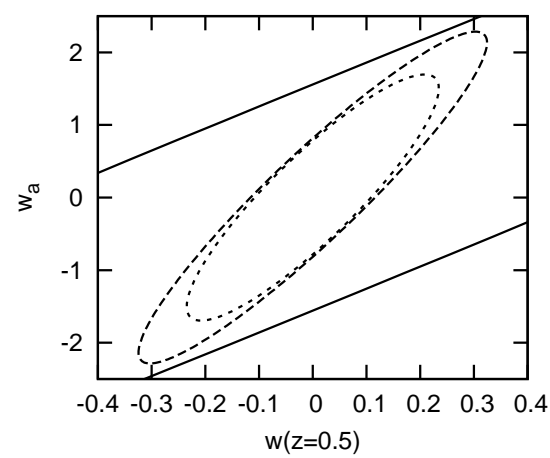

$\mathrm{CMB}+\mathrm{WL}+\mathrm{GW}(\mathrm{AVE})$
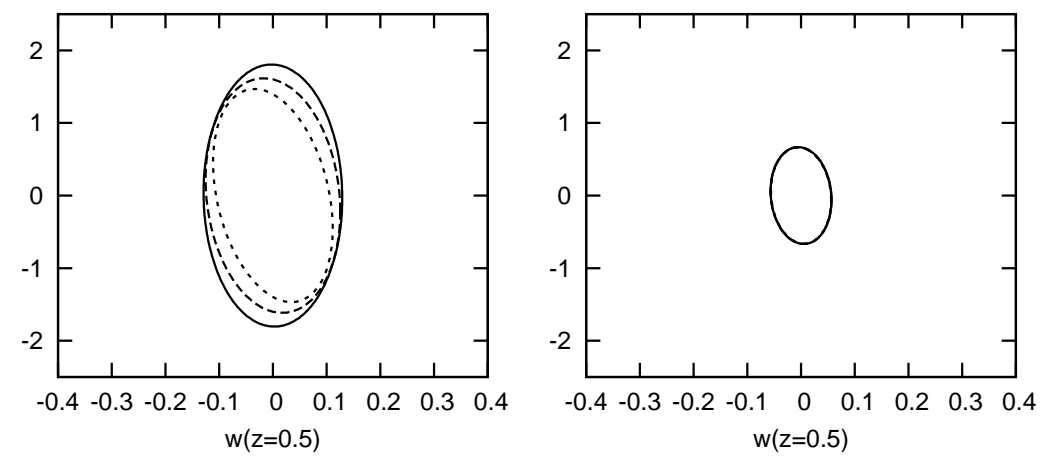

FIG. 6: The constraints on the $\left(w_{0}, w_{a}\right)$ model. The solid ellipses are the forecast $68 \%$ confidence contours $\left(\Delta \chi^{2}=2.28\right)$ for Planck plus the indicated cosmological probe (SN, WL, or BAO) at Stage III using the FoMSWG Fisher matrices [24]. The dashed and dotted ellipses incorporate LISA constraints assuming either 10 usable sources at $z<3$ (dashed) or 30 sources (dotted). The upper panels shows results using the centroiding technique (CEN), while the bottom panels use flux averaging (AVE). The horizontal axis is plotted as $w(z=0.5)=w_{0}+\frac{1}{3} w_{a}$ instead of $w_{0}$ in order to avoid long diagonal contours; note that this transformation preserves the areas of the ellipses.

there are potential sources of systematic error. Some of these, such as strain calibration, coherent peculiar velocities at low redshift [37, 38], and host redshift misidentification, exist irrespective of the method used to estimate the true $D(z)$ from a collection of weakly lensed GW sources with their apparent fluxes and redshifts. However, the issue of uncertainties in the magnification PDF is worth discussing here. It may seem at first glance that the flux-averaging technique is more robust than the centroiding technique described here, because it relies only on the flux conservation constraint $\int_{0}^{\infty} P(\mu) d \mu=1$. This may not be the case for three reasons. One is that in order to remove misidentified host galaxies, it is likely that a BBO-type mission would reject outliers from the $D(z)$ relation [5]. This outlier rejection would eliminate the the tails of the magnification distribution with $|\ln \mu|>(0.4 \ln 10) \Delta M$, where $\Delta M$ is the half-width of the cut in magnitudes. Since $P(\mu)$ is highly asymmetric, with the large- $\mu$ tail much stronger than the small$\mu$ tail, it follows that outlier rejection will result in the conditional probability $\int_{0}^{\infty} P(\mu \mid$ accept $) d \mu<1$ and hence give a positive bias in $D(\mu)$. This can be corrected, but it requires knowledge of the contribution to $\int_{0}^{\infty} P(\mu) d \mu$ from the strong-magnification tail. A second reason has to do with strong lensing: flux conservation implies that the magnification satisfying $\int_{0}^{\infty} P(\mu) d \mu=1$ is the total magnification of all of the images. However, since stronglens time delays are often measured in months (and even longer if the strongly de-magnified central image is significant), it is likely that for many BBO sources there will be additional images whose time delay places them outside the BBO mission lifetime. Finally, in obtaining a successful host redshift (or identifying a source with the correct host rather than another object nearby on the sky), there is likely to be a bias in favor of brighter sources, which results in a success probability that has some dependence on the magnification, $P$ (success) $\sim \mu^{\beta}$. The presence of lensing dispersion then results in a bias in the mean flux of $\sim \beta \operatorname{Var} \mu$; since $\operatorname{Var} \mu$ is of order $10^{-2}$ at $z \sim 1$, such biases will likely be far above the BBO statistical errors, and will have to be corrected using knowledge of $P(\mu)$. Therefore, even the flux-averaging method is sensitive to the particular distribution $P(\mu)$. The problem of how well $P(\mu)$ can be determined via theory (particularly in the presence of baryonic effects), or reduced to a parametric model whose parameters can be simultaneously fit using BBO, is left to future work.

In this paper, we have not attempted to reduce the 
lensing dispersion by using external information, i.e. only the shape of $P(\mu)$ was assumed. The main external sources of information that are commonly considered are weak lensing shear maps and galaxy maps. In principle the way one would incorporate this information would be to write a conditional probability density, e.g. $P(\mu \mid g)$, where $g$ represents the galaxy density field. Galaxy maps have been shown to be helpful because they contain information about the small scales that dominate the lensing variance 13 15]; however the conditional probability distribution $P(\mu \mid g)$ may be very hard to determine theoretically at the required precision. For example, despite recent advances in determining the relation between galaxy luminosity and host halo mass (a key quantity of interest if one is trying to infer the matter density field from galaxy observations) using clustering and lensing data [39, 40], a measurement of the scatter in this relation is not yet possible, and the full distribution of this scatter - required if one is going to compute $P(\mu \mid g)$ - is woefully underconstrained. Nevertheless, for a mission such as LISA that may have only a limited number of usable sources and hence may be dominated by statistical errors due to weak lensing, this may be a useful approach. The weak lensing field is sensitive only to the matter distribution, and so one could imagine that it would be profitable to utilize the smoothed convergence field, $\kappa_{\mathrm{sm}}$, and attempt to centroid the conditional density $P\left(\mu \mid \hat{\kappa}_{\mathrm{sm}}\right)$. WL has traditionally been viewed as not useful for de-lensing of GW sources because most of the lensing variance comes from small scales where weak lensing measurements are noisy [12]. This conclusion should be revisited in future work using the centroiding technique; in particular, this small-scale structure contributes strongly to the high-magnification tail of $P\left(\mu \mid \hat{\kappa}_{\mathrm{sm}}\right)$, and it is not yet known what happens to the Fisher information, which depends largely on the width of the peak of $P\left(\mu \mid \hat{\kappa}_{\mathrm{sm}}\right)$.

\section{Acknowledgements}

We acknowledge useful conversations with Éanna Flanagan and Samaya Nissanke.

C.H. is supported by the U.S. Department of Energy (DE-FG03-92-ER40701), the National Science Foundation (AST-0807337), and the Alfred P. Sloan Foundation. D.H. acknowledges support from the LDRD program at LANL. C.C.'s work was carried out at the Jet Propulsion Laboratory, California Institute of Technology, under contract to the National Aeronautics and Space Administration. He acknowledges support from a JPL Research and Technology Development grant, as well as support from NASA grant NNX07AM80G.

Copyright 2010. All rights reserved.

\section{Appendix A: Information improvement}

This appendix is dedicated to proving the informationbounding inequality:

$$
\left[I_{\ln D^{2}}^{(1)}\right]^{-1 / 2} \leq \sigma_{\mu}
$$

for any distribution such that $\langle\mu\rangle=1$. We also show that equality holds only for the $\Gamma$-distribution:

$$
P(\mu)=\frac{\alpha^{\alpha}}{\Gamma(\alpha)} \mu^{\alpha-1} e^{-\alpha \mu} .
$$

We begin by considering the functions

$$
f(x)=\left(e^{x}-1\right) \sqrt{P(x)}
$$

and

$$
g(x)=-2 \frac{d}{d x} \sqrt{P(x)} .
$$

We now use the Cauchy-Schwarz inequality, $\left[\int f(x) g(x) d x\right]^{2} \leq\left[\int f^{2}(x) d x\right]\left[\int g^{2}(x) d x\right]$. It is readily apparent that $\sigma_{\mu}^{2}=\int f^{2}(x) d x$ since $\mu=e^{x}$ and $\langle\mu\rangle=1$. We can also see that

$$
\begin{aligned}
\int g^{2}(x) d x & =\int\left[2 \frac{d}{d x} \sqrt{P(x)}\right]^{2} d x \\
& =\int\left[\sqrt{P(x)} \frac{d}{d x} \ln P(x)\right]^{2} d x \\
& =I_{\ln D^{2}}^{(1)}
\end{aligned}
$$

Finally,

$$
\begin{aligned}
\int f(x) g(x) d x & =-2 \int\left(e^{x}-1\right) \sqrt{P(x)} \frac{d}{d x} \sqrt{P(x)} d x \\
& =-\int\left(e^{x}-1\right) \frac{d}{d x} P(x) d x \\
& =\int e^{x} P(x) d x=\langle\mu\rangle=1
\end{aligned}
$$

where in the third equality we have used integration by parts and noted that the surface terms vanish since in order to be normalized the probability distribution must vanish faster than $x^{-1}$ as $x \rightarrow \pm \infty$. The Cauchy-Schwarz inequality then states

$$
1 \leq \sigma_{\mu}^{2} I_{\ln D^{2}}^{(1)},
$$

thereby proving Eq. (A1).

Equality holds if and only if $g(x)=\alpha f(x)$ for some constant $\alpha$. Examining Eqs. (A3) and (A4) shows that equality is thus equivalent to a first-order ordinary differential equation for $\sqrt{P(x)}$,

$$
-2 \frac{d}{d x} \sqrt{P(x)}=\alpha\left(e^{x}-1\right) \sqrt{P(x)}
$$


which has the solution

$$
P(x) \propto \exp \left[-\alpha\left(e^{x}-x\right)\right]
$$

Using $P(\mu)=P(x) d x / d \mu=P(x) / \mu$, we may write this as a function of $\mu$ :

$$
P(\mu) \propto \mu^{\alpha-1} e^{-\alpha \mu}
$$

This equation is easily normalized and is given by
Eq. A2 . By inspection its first moment is indeed $\langle\mu\rangle=1$, and its variance is $\operatorname{Var} \mu=\alpha^{-1}$.

We note that for small variance (large $\alpha$ ), the $\Gamma$ distribution (Eq. A2) approaches a Gaussian. This is a direct consequence of the central limit theorem since the $\Gamma$-distribution is simply the reduced- $\chi^{2}$ distribution, i.e. $\chi^{2} / N_{\text {dof }}$ for $N_{\text {dof }}=2 \alpha$ degrees of freedom, and hence represents the distribution of sample averages of $N_{\text {dof }}$ squared unit Gaussian deviates.
[1] A. G. Riess, A. V. Filippenko, P. Challis, A. Clocchiatti, A. Diercks, P. M. Garnavich, R. L. Gilliland, C. J. Hogan, S. Jha, R. P. Kirshner, et al., Astron. J. 116, 1009 (1998), arXiv:astro-ph/9805201.

[2] S. Perlmutter, G. Aldering, G. Goldhaber, R. A. Knop, P. Nugent, P. G. Castro, S. Deustua, S. Fabbro, A. Goobar, D. E. Groom, et al., Astrophys. J. 517, 565 (1999), arXiv:astro-ph/9812133.

[3] W. M. Wood-Vasey, G. Miknaitis, C. W. Stubbs, S. Jha, A. G. Riess, P. M. Garnavich, R. P. Kirshner, C. Aguilera, A. C. Becker, J. W. Blackman, et al., Astrophys. J. 666, 694 (2007), arXiv:astro-ph/0701041.

[4] B. F. Schutz, Nature (London) 323, 310 (1986).

[5] C. Cutler and D. E. Holz, Phys. Rev. D 80, 104009 (2009), 0906.3752.

[6] J. Wambsganss, R. Cen, G. Xu, and J. P. Ostriker, Astron. J. Lett. 475, L81+ (1997).

[7] D. E. Holz and R. M. Wald, Phys. Rev. D 58, 063501 (1998), arXiv:astro-ph/9708036.

[8] D. E. Holz and E. V. Linder, Astrophys. J. 631, 678 (2005), arXiv:astro-ph/0412173.

[9] N. Dalal, D. E. Holz, S. A. Hughes, and B. Jain, Phys. Rev. D 74, 063006 (2006), arXiv:astro-ph/0601275.

[10] S. Nissanke, S. A. Hughes, D. E. Holz, N. Dalal, and J. L. Sievers, ArXiv e-prints (2009), 0904.1017.

[11] D. E. Holz and S. A. Hughes, Astrophys. J. 629, 15 (2005), arXiv:astro-ph/0504616.

[12] N. Dalal, D. E. Holz, X. Chen, and J. A. Frieman, Astron. J. Lett. 585, L11 (2003), arXiv:astro-ph/0206339.

[13] C. Gunnarsson, T. Dahlén, A. Goobar, J. Jönsson, and E. Mörtsell, Astrophys. J. 640, 417 (2006), arXiv:astro$\mathrm{ph} / 0506764$.

[14] J. Jönsson, A. Goobar, and E. Mörtsell, Astrophys. J. 658, 52 (2007), arXiv:astro-ph/0611334.

[15] J. Jönsson, E. Mörtsell, and J. Sollerman, Astron. Astrophys. 493, 331 (2009), 0810.4329.

[16] D. M. Goldberg and D. J. Bacon, Astrophys. J. 619, 741 (2005), arXiv:astro-ph/0406376.

[17] C. Shapiro, D. J. Bacon, M. Hendry, and B. Hoyle, Mon. Not. R. Astron. Soc. pp. 245-+ (2010), 0907.3635.

[18] C. Shang and Z. Haiman, ArXiv e-prints (2010), 1004.3562 .

[19] J. F. Navarro, C. S. Frenk, and S. D. M. White, Astrophys. J. 490, 493 (1997), arXiv:astro-ph/9611107.

[20] R. K. Sheth and G. Tormen, Mon. Not. R. Astron. Soc. 308, 119 (1999), arXiv:astro-ph/9901122.

[21] Y. Wang, D. E. Holz, and D. Munshi, Astron. J. Lett. 572, L15 (2002), arXiv:astro-ph/0204169.
[22] A. Cooray, D. Huterer, and D. E. Holz, Physical Review Letters 96, 021301 (2006), arXiv:astro-ph/0509581.

[23] P. Astier, J. Guy, N. Regnault, R. Pain, E. Aubourg, D. Balam, S. Basa, R. G. Carlberg, S. Fabbro, D. Fouchez, et al., Astron. Astrophys. 447, 31 (2006), arXiv:astro-ph/0510447.

[24] A. Albrecht, L. Amendola, G. Bernstein, D. Clowe, D. Eisenstein, L. Guzzo, C. Hirata, D. Huterer, R. Kirshner, E. Kolb, et al., ArXiv e-prints (2009), 0901.0721.

[25] A. Albrecht, G. Bernstein, R. Cahn, W. L. Freedman, J. Hewitt, W. Hu, J. Huth, M. Kamionkowski, E. W. Kolb, L. Knox, et al., ArXiv Astrophysics e-prints (2006), arXiv:astro-ph/0609591.

[26] B. Kocsis, Z. Frei, Z. Haiman, and K. Menou, Astrophys. J. 637, 27 (2006), arXiv:astro-ph/0505394.

[27] C. L. MacLeod and C. J. Hogan, Phys. Rev. D 77, 043512 (2008), 0712.0618.

[28] R. N. Lang and S. A. Hughes, Astrophys. J. 677, 1184 (2008), 0710.3795.

[29] C. Van Den Broeck, M. Trias, B. S. Sathyaprakash, and A. M. Sintes, ArXiv e-prints (2010), 1001.3099.

[30] S. A. Hughes and D. E. Holz, Classical and Quantum Gravity 20, 65 (2003), arXiv:astro-ph/0212218.

[31] K. G. Arun, S. Babak, E. Berti, N. Cornish, C. Cutler, J. Gair, S. A. Hughes, B. R. Iyer, R. N. Lang, I. Mandel, et al., Classical and Quantum Gravity 26, 094027 (2009), 0811.1011.

[32] J. N. Bode and E. Phinney, in Bulletin of the American Astronomical Society (2009), vol. 41 of Bulletin of the American Astronomical Society, pp. 341-+.

[33] Z. Lippai, Z. Frei, and Z. Haiman, Astron. J. Lett. 676, L5 (2008), 0801.0739.

[34] L. R. Corrales, Z. Haiman, and A. MacFadyen, ArXiv e-prints (2009), 0910.0014.

[35] M. Milosavljević and E. S. Phinney, Astron. J. Lett. 622, L93 (2005), arXiv:astro-ph/0410343.

[36] P. Chang, L. E. Strubbe, K. Menou, and E. Quataert, ArXiv e-prints (2009), 0906.0825.

[37] A. Cooray and R. R. Caldwell, Phys. Rev. D 73, 103002 (2006), arXiv:astro-ph/0601377.

[38] L. Hui and P. B. Greene, Phys. Rev. D 73, 123526 (2006), arXiv:astro-ph/0512159.

[39] I. Zehavi, Z. Zheng, D. H. Weinberg, J. A. Frieman, A. A. Berlind, M. R. Blanton, R. Scoccimarro, R. K. Sheth, M. A. Strauss, I. Kayo, et al., Astrophys. J. 630, 1 (2005), arXiv:astro-ph/0408569.

[40] R. Mandelbaum, U. Seljak, G. Kauffmann, C. M. Hirata, and J. Brinkmann, Mon. Not. R. Astron. Soc. 368, 715 
(2006), arXiv:astro-ph/0511164.

[41] http://lisa.jpl.nasa.gov/

[42] http://jdem.gsfc.nasa.gov/

[43] http://www.lsst.org/lsst 